\title{
Continuidad en la prevención del accidente cerebrovascular en pacientes con fibrilación auricular en la práctica clínica: “Curvas de aprendizaje", nuevos desafíos y necesidades insatisfechas a través del mundo
}

\author{
The Continuum of Stroke Prevention in Patients with Atrial Fibrillation in Clinical Practice: \\ "Learning Curves", New Challenges and Unmet Needs Across the Globe \\ MIROSLAV MIHAJLOVIC ${ }^{1,2}{ }^{\circ}$, TATJANA S. POTPARA $^{1,2}$
}

La prevención del accidente cerebrovascular (ACV) es el pilar fundamental del manejo de pacientes con fibrilación auricular (FA). A partir del momento en que ensayos clínicos históricos demostraran la eficacia de los antagonistas de la vitamina $\mathrm{K}(\mathrm{AVKs})$ en reducir el ACV y la mortalidad en comparación con un tratamiento control (mayormente aspirina o placebo) en pacientes con FA, (1) el panorama de la prevención del ACV en la FA se ha modificado constantemente.

La campaña promoviendo la importancia de una prevención adecuada del ACV isquémico cardioembólico utilizando AVKs en pacientes con AF resultó en el uso generalizado relativamente alto de anticoagulación para la prevención de ACV en FA, (2-4) pero hubo lecciones valiosas que aprender en el camino. Primero, los médicos tratantes debieron aprender que usar aspirina en lugar de anticoagulantes orales era más dañino que útil en los pacientes con FA, dadas tasas comparables de sangrado severo y un efecto marginal de la aspirina sobre la prevención del ACV y la mortalidad. Luego, fue necesario mejorar el uso de la anticoagulación oral para incluir pacientes con FA con uno o más factores de riesgo de $\mathrm{ACV}$, y no aquellos con riesgo de ACV realmente bajo que no necesitan terapia preventiva. Mientras la decisión de usar o no terapia de anticoagulación oral debería basarse en el riego de ACV del paciente individual, el riesgo de sangrado debe ser evaluado para tratar factores de riesgo de sangrado modificables y programar un seguimiento más frecuente de pacientes con factores de riesgo no modificables (por ej: pacientes ancianos, aquellos con antecedentes de sangrado, etc.). Especialmente, los médicos no deberían pasar por alto la naturaleza dinámica del ACV individual y los riesgos de sangrado, que cambian a lo largo del tiempo y necesitan ser reevaluados regularmente.

Existen numerosas limitaciones bien conocidas de los AVKs que plantean un problema importante acerca de su uso óptimo a largo plazo. Los anticoagulantes orales no antagonistas de la vitamina K (NACOs) son al menos tan efectivos como los AVKs, pero más seguros en términos de ACV hemorrágico y sangrado intracraneal (5) y más convenientes para su uso a largo plazo (dosis fija, efecto anticoagulante dependiente de la dosis predecible, menos interacciones droga-droga y ninguna interacción con alimentos). Luego de la aprobación del primer NACO (dabigatrán, en 2010), se han organizado grandes registros internacionales para brindar información acerca de la administración de NACOs y su efectividad y seguridad por fuera de los ensayos clínicos aleatorizados, en el llamado 'mundo real'. $(6,7)$

En este número de la Revista Argentina de Cardiología, Dubner y colaboradores (8) comunicaron hallazgos de la Fase II del Registro Global sobre el Tratamiento Antitrombótico Oral a Largo Plazo en Pacientes con Fibrilación Auricular (GLORIA-AF). Este es un registro prospectivo, multicéntrico, internacional, para estudiar la seguridad y efectividad a largo plazo del dabigatrán en pacientes adultos con FA no valvular recientemente diagnosticada y al menos un factor de riesgo de ACV. El registro GLORIA-AF tiene un diseño de tres fases que incluye la Fase I, antes de la aprobación del dabigatrán; la Fase II, un estudio transversal que comenzó después de la aprobación del dabigatrán y comprendió un seguimiento de 2 años, y la Fase III, que se inició luego de obtenerse la comparabilidad entre AVKs y dabigatrán, con un seguimiento de 3 años (desde 2014 a 2017).

En el estudio de Dubner y colaboradores, (8) que incluyó 15.308 pacientes de 44 países, se documentaron mejorías consistentes con la prescripción de terapia anticoagulante oral en pacientes con FA y riesgo elevado de ACV, particularmente entre los centros europeos, así como el incremento del uso de NACOs, especialmente 


\begin{tabular}{|lccc|}
\hline & $\begin{array}{c}\text { Pacientes con } \\
\text { eventos }\end{array}$ & PA & $\begin{array}{c}\text { Tl cruda por 100 PA } \\
\text { (IC 95\%) }\end{array}$ \\
\hline Mortalidad global & 179 & 7,215 & $2,48(2,13-2,87)$ \\
\hline Accidente cerebrovascular & 47 & 7,192 & $0,65(0,48-0,87)$ \\
\hline Infarto agudo de miocardio & 36 & 7,204 & $0,50(0,35-0,69)$ \\
\hline Sangrado mayor & 70 & 7,199 & $0,97(0,46-0,84)$ \\
\hline
\end{tabular}

Tabla. Tasas de incidencia cruda a los 2 años de seguimiento en pacientes tratados con dabigatrán

IC: Intervalo de confianza. TI: Tasa de incidencia. PA = Paciente-años.

en Europa y Norteamérica. (8) En comparación con la Fase I, (10) en la Fase II del registro GLORIA-AF el uso total de drogas antiplaquetarias para la prevención de $\mathrm{ACV}$ en pacientes con FA fue significativamente menor, aunque es todavía alto en Asia (25.1\% de los pacientes) y Norteamérica (14.1\%). La profilaxis trombótica inadecuada fue más pronunciada en pacientes con un solo factor de riesgo de ACV (terapia antiplaquetaria en el $10.0 \%$ de los pacientes, y sin tratamiento antitrombótico en el 6.8\%), en comparación con aquellos con dos o más factores de riesgo de ACV (19.3\% y $14.1 \%$, respectivamente). La alta tasa de prescripción de aspirina en Norteamérica puede ser explicada por la recomendación de las guías locales en el momento en que se estaba llevando a cabo la Fase II del GLORIA$\mathrm{AF}$, mientras que las tasas altas de uso de aspirina y las bajas tasas de prescripción de anticoagulantes orales (55.2\%) en Asia podrían haber estado influenciadas por la estimación de alto riesgo de sangrado. Los resultados de los registros en curso, $(9,11)$ mostrarán las tendencias más recientes en la prevención de ACV en la práctica clínica contemporánea.

La comunicación de Dubner y colaboradores (8) trató también la importante dificultad en el tratamiento con NACOs a largo plazo. A los 24 meses, el 30\% de los pacientes no estaban recibiendo dabigatrán (a pesar de una tasa de abandono significativamente menor en comparación con AVKs), y el problema de mantener la persistencia en la administración de NACOs a largo plazo continúa siendo un desafío para los médicos clínicos. En la Fase II del registro GLORIA-AF, la mayor parte del abandono ocurrió tempranamente una vez iniciada la terapia. Los motivos más comunes de abandono no estuvieron relacionados a eventos adversos, sino a "otra(s)" razón(es) sin explicación ulterior. Sería muy informativo un análisis detallado de los motivos de abandono, ya que potencialmente revelaría áreas del manejo de pacientes que pueden mejorarse aún más.

Finalmente, las tasas de eventos adversos observadas en el estudio de Dubner y colaboradores (8) (ver Tabla) son un amplio reflejo de los efectos del dabigatrán en el ensayo fundamental aleatorizado versus warfarina para la prevención de ACV en FA. (12) Además, los hallazgos de la Fase II del registro GLORIA-AF concuerdan con otros reportes del mundo real que señalan tasas decrecientes de ACV y tasas aceptables de sangrado con aumento en el uso de terapia anticoagulante oral. (13) No obstante, como ha sido observado en otras series de datos, las tasas de mortalidad global en pacientes con FA permanecen considerablemente altas, lo que sugiere que se deben identificar a tiempo otros elementos tales como factores de riesgo cardiovascular y comorbilidades concomitantes y reconocerlos como parte del manejo holístico integral de los pacientes con FA. (14)

\section{Declaración de conflicto de intereses}

Los autores declaran que no poseen conflicto de intereses.

(Véanse formularios de conflicto de intereses de los autores en la web/ Material suplementario).

\section{Consideraciones éticas}

No aplican

\section{BIBLIOGRAFÍA}

1. Hart RG, Pearce LA, Aguilar MI. Meta-analysis: antithrombotic therapy to prevent stroke in patients who have nonvalvular atrial fibrillation. Ann Intern Med. 2007;146(12):857-67.

2. Potpara TS, Dan GA, Trendafilova E, Goda A, Kusljugic Z, Manola $\mathrm{S}$, et al. Stroke prevention in atrial fibrillation and 'real world' adherence to guidelines in the Balkan Region: The BALKAN-AF Survey. Sci Rep. 2016;6:20432.

3. Hsu JC, Akao M, Abe M, Anderson KL, Avezum A, Glusenkamp N, et al. International Collaborative Partnership for the Study of Atrial Fibrillation (INTERAF): Rationale, Design, and Initial Descriptives. J Am Heart Assoc. 2016;5(11).

4. Boriani G, Proietti M, Laroche C, Fauchier L, Marin F, Nabauer M, et al. Contemporary stroke prevention strategies in 11096 European patients with atrial fibrillation: a report from the EURObservational Research Programme on Atrial Fibrillation (EORP-AF) Long-Term General Registry. Europace. 2018;20(5):747-57.

5. Ruff CT, Giugliano RP, Braunwald E, Hoffman EB, Deenadayalu N, Ezekowitz MD, et al. Comparison of the efficacy and safety of new oral anticoagulants with warfarin in patients with atrial fibrillation: a meta-analysis of randomised trials. Lancet. 2014;383(9921):955-62. 6. Potpara TS, Lip GY. Postapproval Observational Studies of NonVitamin K Antagonist Oral Anticoagulants in Atrial Fibrillation. JAMA. 2017;317(11):1115-6.

7. Gumprecht J, Lip GYH, Potpara TS. Regional registries on the management of atrial fibrillation: Essential pieces in the global puzzle. Int J Cardiol Heart Vasc. 2020;26:100473.

8. Sergio Dubner GYL, Christine Teusch, Hans Christian Diener, Cecilia Zeballos, Jonathan L Halperin, Chang Sheng Ma, Miney Paquette, Menno V. Huisman. Stroke Prevention in Atrial Fibrillation. Findings from the GLORIA-AF Registry. Revista Argentina de Cardiología. 2020. 
9. Huisman MV, Lip GY, Diener HC, Dubner SJ, Halperin JL, Ma CS, et al. Design and rationale of Global Registry on Long-Term Oral Antithrombotic Treatment in Patients with Atrial Fibrillation: a global registry program on long-term oral antithrombotic treatment in patients with atrial fibrillation. Am Heart J. 2014;167(3):329-34. 10. Huisman MV, Ma CS, Diener HC, Dubner SJ, Halperin JL, Rothman KJ, et al. Antithrombotic therapy use in patients with atrial fibrillation before the era of non-vitamin $\mathrm{K}$ antagonist oral anticoagulants: the Global Registry on Long-Term Oral Antithrombotic Treatment in Patients with Atrial Fibrillation (GLORIA-AF) Phase I cohort. Europace. 2016;18(9):1308-18.

11. Potpara TS, Lip GYH, Dagres N, Crijns H, Boriani G, Kirchhof P, et al. Cohort profile The ESC EURObservational Research Programme Atrial Fibrillation III (AF III) Registry. Eur Heart J Qual Care Clin Outcomes. 2020.

12. Connolly SJ, Ezekowitz MD, Yusuf S, Eikelboom J, Oldgren J, Parekh A, et al. Dabigatran versus warfarin in patients with atrial fibrillation. N Engl J Med. 2009;361(12):1139-51.

13. Boriani G, Proietti M, Laroche C, Fauchier L, Marin F, Nabauer $\mathrm{M}$, et al. Association between antithrombotic treatment and outcomes at 1-year follow-up in patients with atrial fibrillation: the EORP-AF General Long-Term Registry. Europace. 2019;21(7):1013-22.

14. Lip GYH. The ABC pathway: an integrated approach to improve AF management. Nat Rev Cardiol. 2017;14(11):627-8. 